Document downloaded from:

http://hdl.handle.net/10251/134685

This paper must be cited as:

Coves, A.; Torregrosa-Penalva, G.; Vicente, C.; Gimeno, B.; Boria Esbert, VE. (2008). Multipactor discharges in parallel-plate dielectric-loaded waveguides including space-charge effects. IEEE Transactions on Electron Devices. 55(9):2505-2511. https://doi.org/10.1109/TED.2008.927945

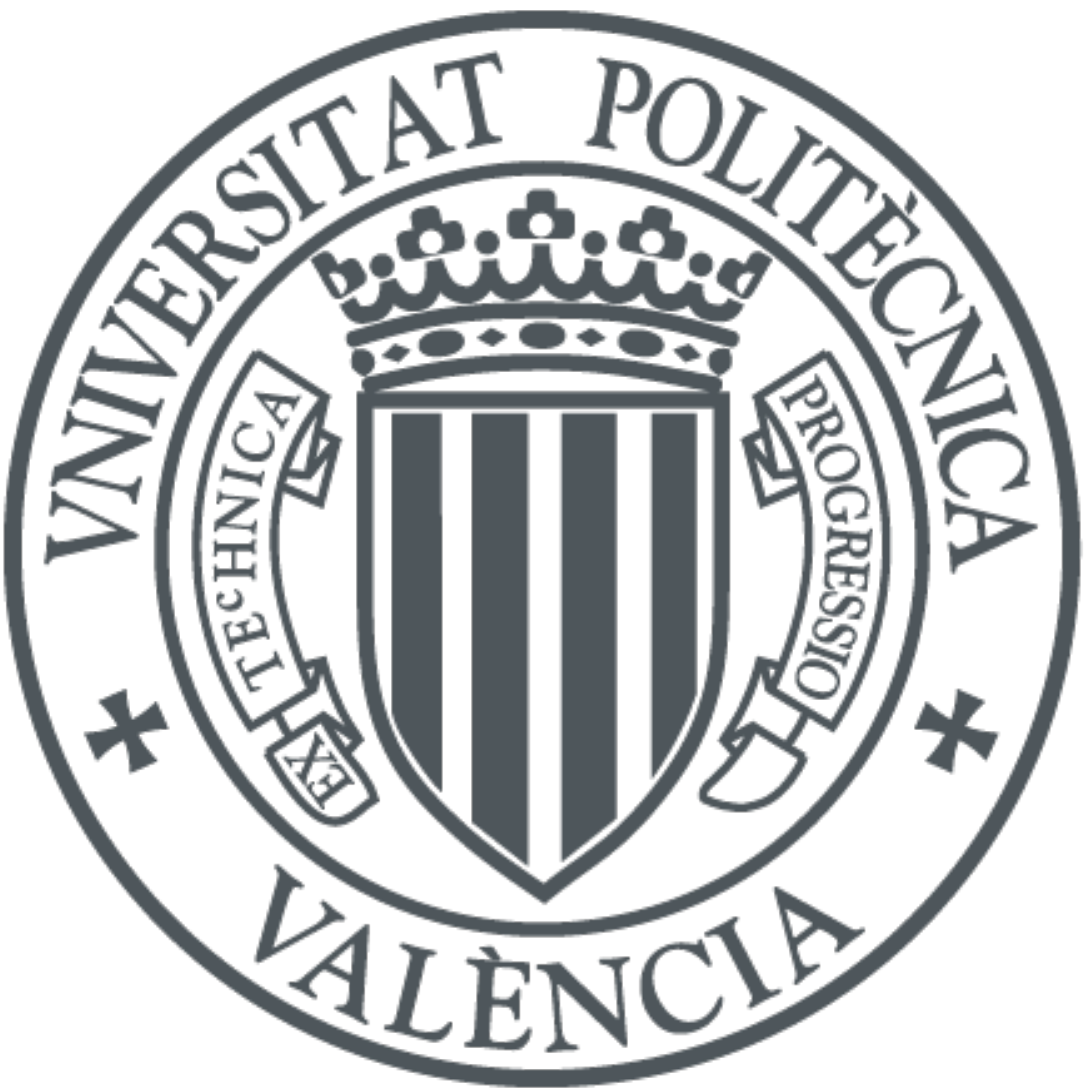

The final publication is available at

https://doi.org/10.1109/TED.2008.927945

Copyright Institute of Electrical and Electronics Engineers

Additional Information 


\title{
Multipactor Discharges in Parallel-Plate Dielectric-Loaded Waveguides Including Space-Charge Effects
}

\author{
Ángela Coves, Member, IEEE, Germán Torregrosa-Penalva, Member, IEEE, Carlos Vicente, \\ Benito Gimeno, Member, IEEE, and Vicente E. Boria, Senior Member, IEEE
}

\begin{abstract}
The objective of this paper is to study the time evolution of multipactor discharges in a parallel-plate dielectric-loaded waveguide. The electron discharge phenomena investigated in this paper include the effects of space charge, as well as the presence of time varying static fields. In order to perform the simulations presented in this paper, an approach considering simultaneously multiple effective electrons has been used. The results obtained demonstrate that multipactor discharges do turn off by themselves under certain circumstances when they occur in such dielectric-loaded structures.
\end{abstract}

Index Terms-Dielectric, multipactor discharge, space charge, static field.

\section{INTRODUCTION}

$\mathbf{T}$ HE multipactor effect is a resonant vacuum electron discharge that can occur in microwave and millimeterwave subsystems, such as filters, multiplexers, and RF satellite payloads. These components usually include dielectric materials employed as resonators and supporting elements. Most publications in recent years study the multipactor problem in microwave devices without dielectric materials, as in [1]-[7], where the multipactor breakdown voltage threshold is studied, thus leading to the construction of generalized susceptibility diagrams. On the other hand, other studies on multipactor discharges on dielectric windows [8]-[10] have focused on both the conditions for multipactor initiation, as well as on the evolution of the discharge, and its saturation mechanism due to the surface charging of the dielectric, using a dynamic theory. Indeed, the multipactor problem in microwave devices including dielectric materials dealing with special geometries has been studied in few publications [11]-[13]. The multipactor effect in a two-surface regime, including the effects of dielectric materials, has been investigated in a previous work

Manuscript received March 13, 2008. This work was supported by the Spanish Government's Ministerio de Educación y Ciencia under Project TEC2007-67630-C03-01. The review of this paper was arranged by Editor W. Menninger.

Á. Coves and G. Torregrosa-Penalva are with the Área de Teoría de la Señal y Comunicaciones, Universidad Miguel Hernández, 03202 Elche, Spain (e-mail: gtorregrosa@umh.es).

C. Vicente is with Aurorasat Software and Testing, Parc Cientific Universitat de València, 46980 Paterna, Spain (e-mail: carlos.vicente@aurorasat.es).

B. Gimeno is with the Departamento de Física Aplicada y Electromagnetismo and the Instituto de Ciencia de Materiales, Universidad de Valencia, 46100 Burjassot, Spain (e-mail: benito.gimeno@uv.es).

V. E. Boria is with the Departamento de Comunicaciones, Universidad Politécnica de Valencia, 46022 Valencia, Spain (e-mail: vboria@dcom.upv.es). of the authors [14] for a particular parallel-plate dielectricloaded waveguide, using the traditional single effective electron model, and without considering space-charge effects. In the specific case studied in that work, the dielectric layer charges negatively, allowing a negative static field to build up. This dc field eventually leads to a single-surface multipactor from the metallic surface before the electron discharge is finally turned off.

We have extended the analysis of the previous work by studying the time evolution of multipactor discharges in a parallel-plate dielectric-loaded waveguide, including the effects of space charge, and considering the simultaneous tracking of multiple effective electrons.

In this paper, we assume a Rayleigh probability density function for the departure kinetic energy of the emitted electrons. The dynamics of each effective electron are solved exactly, conditioned by the external RF electric field, the dc electric field appearing because of the charging of the dielectric surface, and the repulsion of the charge density existing between the plates. The proposed technique allows the examination of different geometries under a variety of working conditions. We will discuss the effects of the appearance of the dc electric field and the space-charge effect during the time evolution of the discharge.

Section II describes the physical model used in our simulations. In Section III, we show the results obtained for a multipactor discharge occurring in an empty silver parallel-plate waveguide. In this section, the same problem in a parallel-plate dielectric-loaded waveguide is also analyzed. A few concluding remarks are made in Section IV.

\section{THEORY}

The geometry and dimensions of the problem under investigation are given in Fig. 1. An RF voltage swing of the form $V(t)=V_{0} \cos (\omega t+\alpha)$ is applied between both plates, where $V_{0}$ is the amplitude, $\omega=2 \pi f$ where $f$ is the RF frequency, and $\alpha$ is the initial phase at time $t=0 \mathrm{~s}$. An RF electric field associated to this voltage swing appears between both plates. The RF electric field in the gap space $\mathbf{E}$, which is perpendicular to both metallic plates, is given by $\mathbf{E}=E_{0} \cos (\omega t+\alpha) \widehat{\mathbf{x}}$, where $E_{0}$ can be calculated by means of an electrostatic approach as follows:

$$
E_{0}=\frac{V_{0} \varepsilon_{r}}{h+\varepsilon_{r}(d-h)}
$$




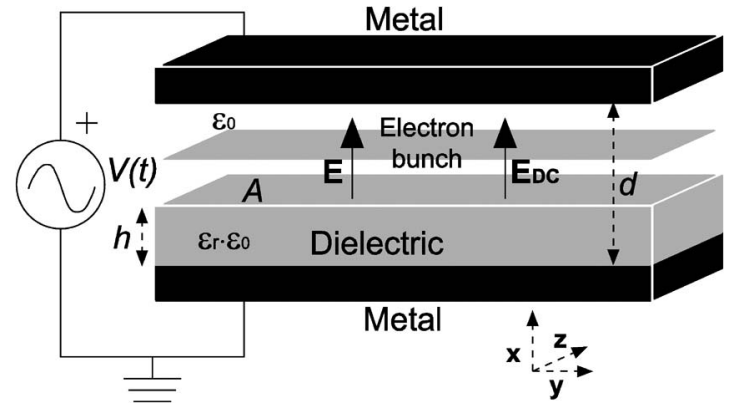

Fig. 1. Parallel-plate waveguide partially filled with a dielectric material of relative permittivity $\varepsilon_{r}$. The distance between the two metallic parallel surfaces is $d$, whereas the dielectric slab, of height $h$, is placed on top of one of the metallic plates.

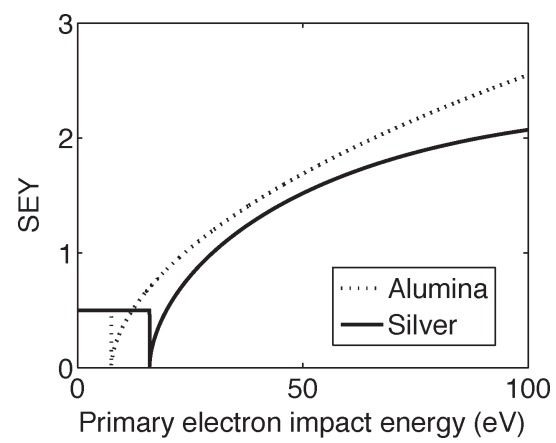

Fig. 2. Secondary electron emission yield curves for silver and alumina extracted from [6] and [9].

where $d$ is the distance between the metallic plates, $h$ is the height of the dielectric sheet, and $\varepsilon_{r}$ is its dielectric permittivity, as shown in Fig. 1. The magnetic field associated to this RF electric field has been neglected, as suggested by many authors [1]-[3], [7], [15]. Due to the characteristics of the geometry under study and the fields present in the gap between the plates, electrons are accelerated in the direction normal to the surfaces. This allows the assumption of the 1-D model approach considered in this paper.

An electron inside the gap space is accelerated by the RF electric field. The movement may eventually lead the electron to impact with any surface. Each collision can result in the emission or absorption of secondary electrons. The number of electrons emitted or absorbed after each impact is determined by the value of the secondary electron yield (SEY) parameter $\delta(\delta>1$ if secondary electrons are emitted, and $\delta<1$ if they are absorbed). The value of parameter $\delta$ is calculated by means of realistic SEY functions that include the effect of reflected electrons for low impact energies of primary electrons, which must be accounted in order to obtain accurate results [6], [16]. The SEY functions used are obtained for the normal incidence of the primary electrons. Fig. 2 shows the SEY function for silver and alumina. The SEY properties for the materials employed in the simulations presented in this paper are defined by the following parameters: the primary electron impact kinetic energies which yield $\delta=1, W_{1}$, and $W_{2}$, the impact energy $W_{\text {MAX }}$ necessary for a primary electron to yield $\delta=\delta_{\mathrm{MAX}}$, which is the maximum value of the SEY function, and the value of the primary electron impact energy $W_{0}(\delta=0)$ that limits the region of elastic collisions. The SEY function used assumes $\delta=0.5$ for low impact energies of primary electrons. This value has been experimentally validated in [17].

In this paper, a modified approach to the study of multipactor discharges using the traditional single effective electron model [18] is employed. Basically, it consists of the simultaneous tracking of the trajectories of $M$ effective electrons. The total number of electrons $N$ at a given time $t$ is calculated by means of the following:

$$
N(t)=\sum_{i=1}^{M} N_{i}(t)
$$

where $N_{i}(t)$ is the population of electrons of the $i$ th effective electron at time $t$. After the $i$ th effective electron impacts at time $t$ with any surface, $N_{i}$ is modified according to the $\delta$ value provided by the SEY function so that

$$
N_{i}(t+\Delta t)=\delta N_{i}(t)
$$

where $\Delta t$ is the time step used in the simulations. It should be emphasized that the $\delta$ function will be, in general, different in the metal and dielectric surfaces. The secondary electron departure kinetic energy $W_{s}$ is assumed to follow a Rayleigh probability density function of the following form:

$$
f\left(W_{s}\right)=\frac{W_{s}}{W_{g}^{2}} \exp \left(\frac{-W_{s}^{2}}{2 W_{g}^{2}}\right)
$$

with parameter $W_{g}=3 \mathrm{eV}$.

When the structure under study includes a dielectric layer, the electrons emitted by this surface charge the dielectric material positively, whereas the electrons absorbed by the dielectric layer charge it negatively. This charge gives rise to a dc electric field. This field is time varying, as it depends on the net charge of the dielectric layer. The dc electric field, after the $i$ th effective electron impacts with the dielectric surface $\mathbf{E}_{\mathrm{dc}}=E_{\mathrm{dc}} \hat{\mathbf{x}}$, can be calculated using the electrostatic approach

$$
E_{\mathrm{dc}}(t+\Delta t)=E_{\mathrm{dc}}(t)+\frac{e N_{i}(t)(\delta-1)}{2 A \varepsilon_{0}}
$$

where $E_{\mathrm{dc}}(t)$ is the value of the dc field before the impact of the $i$ th effective electron with the dielectric surface, $\delta$ is the SEY parameter for the dielectric surface, $-e$ is the charge of an electron, and $A$ is the area of the dielectric surface considered (despite the 1-D assumption of the model, the charge is considered to be uniformly distributed on an area $A$ ). Note that at $t=0, E_{\mathrm{dc}}=0$.

In addition, space-charge effects have been included, following the same procedure introduced in [19]-[21]. This is accomplished by a single electron sheet that (forced by the RF and dc electric fields) moves across the parallel-plate waveguide (this electron sheet is shown in Fig. 1). This electron bunch has an electron density which is given by

$$
\sigma_{b}=-\frac{e N}{A} .
$$

When this sheet impacts on any surface, a new sheet is generated by elastic collision. 
TABLE I

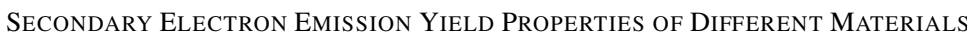

\begin{tabular}{|c|c|c|c|c|c|}
\hline Material & $\mathbf{W}_{\text {MAX }}(\mathbf{e V})$ & $\mathbf{W}_{\mathbf{1}} \mathbf{( e V )}$ & $\mathbf{W}_{\mathbf{2}}(\mathbf{e V})$ & $\mathbf{W}_{\mathbf{0}}(\mathbf{e V})$ & $\delta_{\mathbf{M A X}}$ \\
\hline Silver & 165.0 & 30.0 & 5000.0 & 15.99 & 2.22 \\
\hline Diel $_{\text {silver }}$ & 165.0 & 30.0 & 5000.0 & 15.99 & 2.22 \\
\hline Alumina & 1300.0 & 23.8 & 14135.0 & 7.50 & 6.50 \\
\hline
\end{tabular}

Therefore, the dynamics of each effective electron are conditioned by the RF electric field, the dc electric field (if $h>0$ ), and the repulsion of the electron sheet. The equation of motion of each effective electron across the plates then becomes

$$
E_{0} \cos (\omega t+\alpha)+E_{\mathrm{dc}}+\frac{\sigma_{b}}{2 \varepsilon_{0}} \operatorname{sgn}\left(x_{i}-x_{b}\right)=-\frac{m}{e} \frac{d^{2} x_{i}}{d t^{2}}
$$

where $x_{i}$ is the position of the $i$ th effective electron, $x_{b}$ is the position of the electron bunch, $m$ is the electron mass, and the sign function sgn is defined as

$$
\operatorname{sgn}\left(x_{i}-x_{b}\right)= \begin{cases}+1, & \text { if } x_{i}>x_{b} \\ 0, & \text { if } x_{i}=x_{b} \\ -1, & \text { if } x_{i}<x_{b}\end{cases}
$$

The equation of motion of the electron sheet is as follows:

$$
E_{0} \cos (\omega t+\alpha)+E_{\mathrm{dc}}=-\frac{m}{e} \frac{d^{2} x_{b}}{d t^{2}} .
$$

Solving (7), the trajectory and velocity of each effective electron are obtained analytically as follows:

$$
\begin{aligned}
x_{i}(t)= & x_{0 i}+\left[v_{0 i}+e \frac{E_{0}}{m \omega} \sin \left(\omega t_{0 i}+\alpha\right)\right]\left(t-t_{0 i}\right) \\
& +e \frac{E_{0}}{m \omega^{2}}\left[\cos (\omega t+\alpha)-\cos \left(\omega t_{0 i}+\alpha\right)\right] \\
& -e \frac{E_{\mathrm{dc}}}{2 m}\left(t-t_{0 i}\right)^{2}-e \frac{\sigma_{b}}{4 \varepsilon_{0} m} \operatorname{sgn}\left(x_{i}-x_{b}\right)\left(t-t_{0 i}\right)^{2}
\end{aligned}
$$

$$
\begin{aligned}
v_{i}(t)= & v_{0 i}+\left[e \frac{E_{0}}{m \omega}\left(\sin \left(\omega t_{0 i}+\alpha\right)-\sin (\omega t+\alpha)\right]\right. \\
& -e \frac{E_{\mathrm{dc}}}{m}\left(t-t_{0 i}\right)-e \frac{\sigma_{b}}{2 \varepsilon_{0} m} \operatorname{sgn}\left(x_{i}-x_{b}\right)\left(t-t_{0 i}\right)
\end{aligned}
$$

where $x_{0 i}$ and $v_{0 i}$ are the $i$ th effective electron position and velocity, respectively, at time $t_{0 i}$. Solving (9) yields the trajectory and velocity of the electron sheet

$$
\begin{aligned}
x_{b}(t)= & x_{0 b}+\left[v_{0 b}+e \frac{E_{0}}{m \omega} \sin \left(\omega t_{0 b}+\alpha\right)\right]\left(t-t_{0 b}\right) \\
& +e \frac{E_{0}}{m \omega^{2}}\left[\cos (\omega t+\alpha)-\cos \left(\omega t_{0 b}+\alpha\right)\right] \\
& -e \frac{E_{\mathrm{dc}}}{2 m}\left(t-t_{0 b}\right)^{2} \\
v_{b}(t)= & v_{0 b}+\left[e \frac{E_{0}}{m \omega}\left(\sin \left(\omega t_{0 b}+\alpha\right)-\sin (\omega t+\alpha)\right]\right. \\
& -e \frac{E_{\mathrm{dc}}}{m}\left(t-t_{0 b}\right)
\end{aligned}
$$

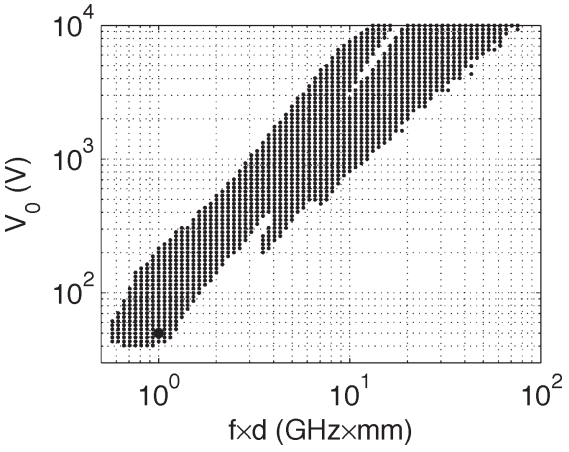

Fig. 3. Susceptibility chart of an empty silver parallel-plate waveguide. Three hundred sixty individual electrons (with different initial phases) have been employed to determine the multipactor regions. The operating point corresponding to $f \times d=1 \mathrm{GHz} \times \mathrm{mm}$ and $V_{0}=50 \mathrm{~V}$ is highlighted.

where $x_{0 b}$ and $v_{0 b}$ are the electron bunch position and velocity, respectively, at time $t_{0 b}$.

Future work on the model suggested in this section might involve the introduction of the second order effects such as extension to a 2-D approximation, inclusion of the RF magnetic field, or consideration of cavity detuning in realistic waveguide devices.

\section{NumericAl Results AND Discussion}

\section{A. Parallel Silver Plate Waveguide}

An algorithm based on the expressions given in Section II has been programmed using Fortran to provide the results outlined next. This section shows the results obtained for a multipactor discharge occurring in an empty silver parallel-plate waveguide with the newly developed simulation tool. The SEY properties of the silver surfaces are given in Table I. To determine $x_{0 i}$, the effective electrons are initially randomly distributed along the $x$ dimension of the waveguide (following a uniform distribution). Their initial velocity $v_{0 i}$ is randomly calculated, assuming the same Rayleigh distribution employed to calculate the secondary electron departure kinetic energy after an impact occurs against one of the silver plates.

In order to check our algorithm, the susceptibility chart for this particular waveguide has been obtained by simulation, using 360 individual electrons, as shown in Fig. 3. In this chart, dark regions indicate the operating conditions that make multipactor discharges to appear. Three hundred sixty single effective electrons (with different initial phases of the RF field) have been employed to determine the multipactor regions. For each working condition, it is considered that multipactor has occurred if $N$ reaches a steady saturating value.

Next, Fig. 4 shows the time evolution of the population of electrons under two different scenarios: In one of them, 


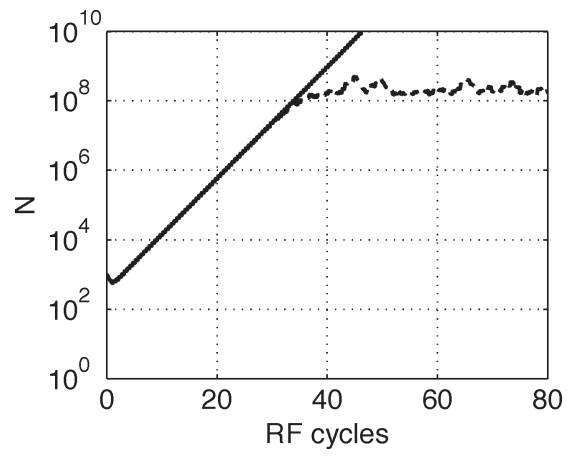

Fig. 4. Time evolution of the number of electrons $N$ (solid line) when spacecharge effects are not considered and (dashed line) when they are included for the empty silver parallel-plate waveguide.

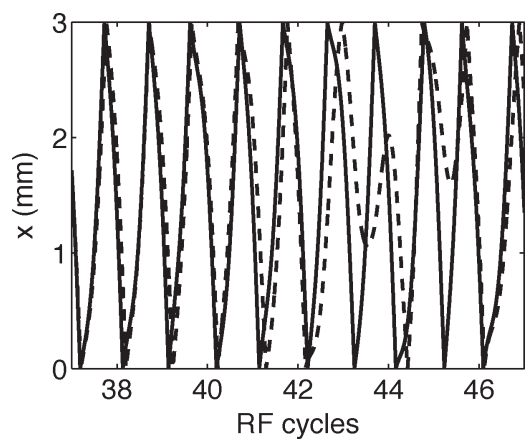

Fig. 5. One-dimensional trajectories of one of the $M=1000$ effective electrons (solid line) when space-charge effects are not considered and (dashed line) when they are included for the empty silver parallel-plate waveguide.

space-charge effects are neglected, whereas in the other one, space-charge effects are considered, as introduced in the previous section. $M=1000$ effective electrons are simultaneously used in order to prevent any single effective electron to become statistically significant in the development of the discharge. The parameters of the geometry and RF signal initial conditions used for all simulations performed throughout this paper (unless otherwise indicated) are as follows: $d=3 \mathrm{~mm}, f=$ $1 / 3 \mathrm{GHz}, V_{0}=50 \mathrm{~V}, \alpha=0^{\circ}$, and $A=10 \mathrm{~cm}^{2}$. The accuracy of the Monte Carlo simulations is guaranteed by selecting a simulation time step of $1 /\left(10^{5} \cdot f\right)$. For the conditions stated earlier, the operating point is inside the multipactor region, as can be observed in Fig. 3. When space-charge effects are not included, there is no saturating mechanism observed in the simulation. Thus, $N$ grows at an exponentially constant rate. In this case, there is no interaction between the $M=1000$ effective electrons being tracked simultaneously; thus, each one of them acts independently of the rest.

When space-charge effects are included, following a similar approach to that presented in [21], $N$ reaches a steady saturating value after approximately $40 \mathrm{RF}$ cycles. In this case, the $M=1000$ effective electrons interact among themselves through the space-charge sheet accounting for space-charge effects. The space-charge saturating effect is well explained by Figs. 5 and 6.

When the electron density is not very high (which, for the situation under study, occurs for $N \sim 3 \times 10^{8}$ ), space-charge effects are basically negligible. The single effective electron follows a typical first-order multipactor trajectory (for the first

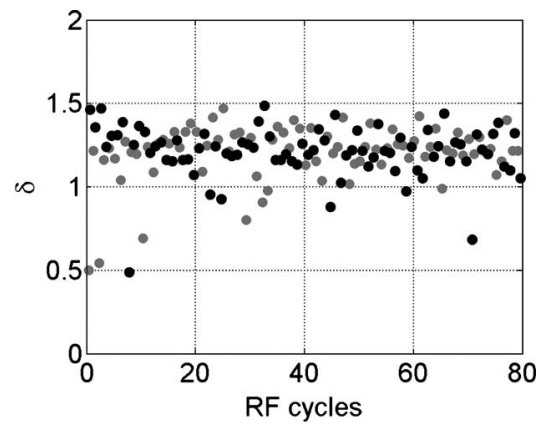

(a)

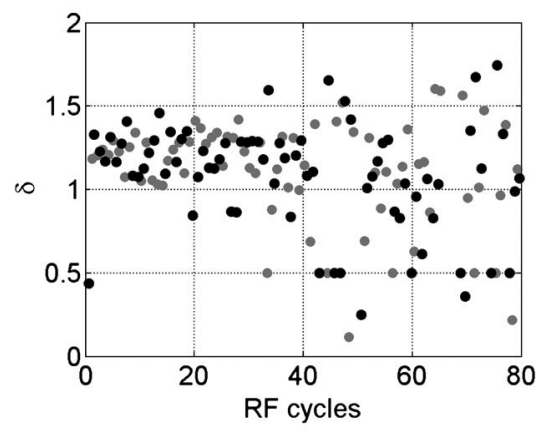

(b)

Fig. 6. Impact energies for collisions against the (black circles) top silver layer and (gray circles) bottom silver layer for one of the $M=1000$ effective electrons in the empty silver parallel-plate waveguide. (a) Space-charge effects are not considered. (b) Space-charge effects are considered.

40 RF cycles), as shown in Fig. 5, when space-charge effects are not considered, as well as when its effects are accounted for. Meanwhile, each effective electron, due to its resonant movement, always impacts against the top and bottom silver layers approximately with the same kinetic energy [yielding an emission of $1<\delta<1.5$ secondary electrons per impact, as can be seen in Fig. 6(a) and (b)]. The kinetic energies of the impacting electrons are near the first crossover $(\delta=1)$ of the SEY functions. According to [22], under these circumstances, the magnetic field can be neglected, as it has been assumed in our model.

Once the population of electrons reaches a significant number $\left(N \sim 3 \times 10^{8}\right.$ in the conditions under study), the effects of space charge become noticeable. Each effective electron is unable to keep up with its previous synchronization (for the dashed effective electron trajectory shown in Fig. 5, this happens around RF cycle number 43). The space-charge bunch is capable of repelling the effective electrons whenever they are close together, modifying their trajectories. This causes the electrons to collide with the top and bottom silver layers much sooner or later than the instants when the RF electric field changes its sign. In turn, this implies low impact energy collisions, so that the metallic plates absorb electrons (as can be observed in Fig. 6(b) from RF cycle 40). The reduction in $N$ automatically weakens the strength of the electron bunch, so that its effects on the effective electrons are softer. Effective electrons are no longer strongly repelled by the electron bunch, which allows $N$ to recover again and the process starts all over again, reaching the saturation state observed in Fig. 4. 


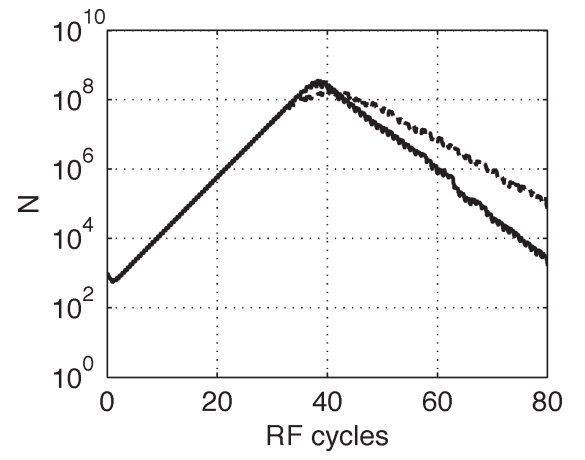

Fig. 7. Time evolution of the number of electrons $N$ (solid line) when spacecharge effects are not considered and (dashed line) when they are included for the silver parallel-plate diel silver $_{\text {loaded waveguide. }}$

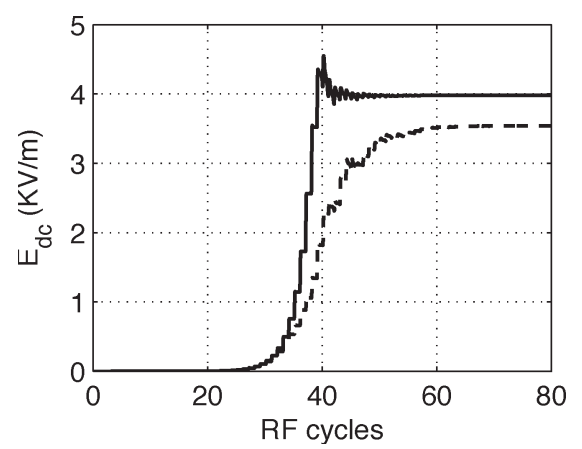

Fig. 8. $\quad E_{\mathrm{dc}}$ static field time evolution (solid line) when space-charge effects are not considered and (dashed line) when they are included for the silver parallel-plate diel $\mathrm{s}_{\text {silver }}$ loaded waveguide.

\section{B. Parallel-Plate Silver-Like Dielectric-Loaded Waveguide}

The situations presented in the previous section are repeated, introducing a thin dielectric layer on top of the bottom silver surface for studying the effects of a time varying $E_{\mathrm{dc}}$ static field. In order to facilitate the comparison between both the free space and the dielectric-loaded waveguides, a fictitious dielectric material with the same SEY properties as silver is used to load the waveguide (see material diel silver $_{\text {in Table I). }}$ For the cases simulated in this section, we used $h=3 \mu \mathrm{m}$ and $\epsilon_{r}=9.5$.

Fig. 7 shows the time evolution of $N$ for the silver parallelplate dielectric-loaded waveguide. The main difference between the responses observed in Figs. 4 and 7 is the fact that, in the latter case, the discharges eventually switch off by themselves (similarly to the behavior described in [14]), as a consequence of the dielectric presence.

The extinction of the multipactor discharges for the particular operating conditions being studied is due to the appearance of a time varying static field whose time evolution is shown in Fig. 8. The $E_{\mathrm{dc}}$ static field causes effective electrons to lose their synchronized first-order multipactor trajectory, as evident from the trajectory of one of the $M=1000$ effective electrons used in the simulations shown in Fig. 9. The explanation of what is happening to the development of the multipactor discharge can be better stated with the help of Fig. 10(a) and (b).

For the first $40 \mathrm{RF}$ cycles, both the $E_{\mathrm{dc}}$ static field and space-charge effects are very weak, and effective electrons follow a first-order multipactor trajectory (as can be observed in Fig. 9). Effective electrons impact against the top metallic layer

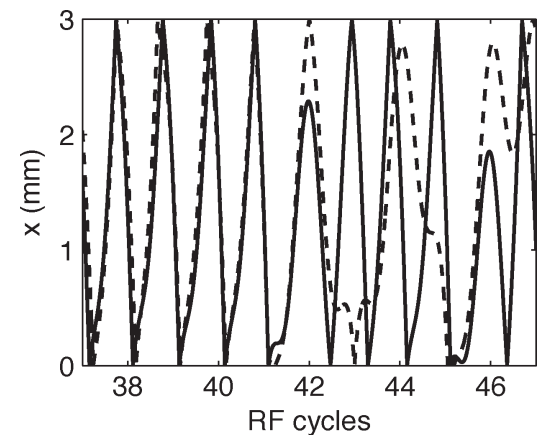

Fig. 9. One-dimensional trajectories of one of the $M=1000$ effective electrons (solid line) when space-charge effects are not considered and (dashed line) when they are included for the silver parallel-plate diel $_{\text {silver }}$ loaded waveguide.

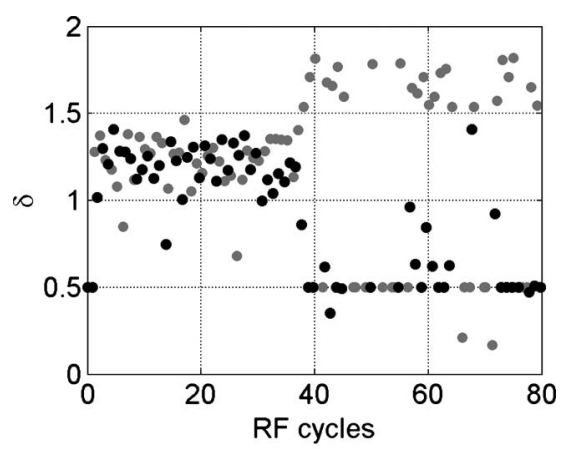

(a)

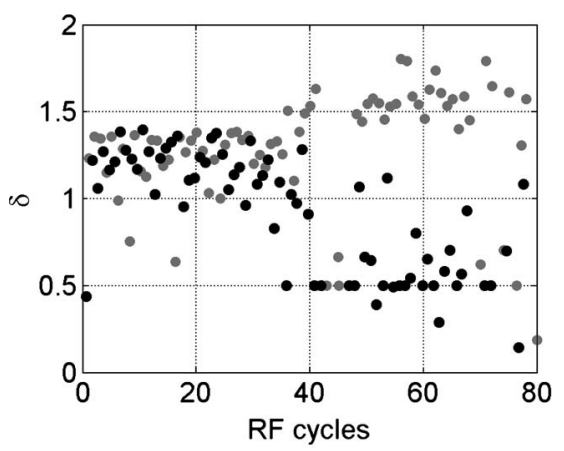

(b)

Fig. 10. Impact energies for collisions against the (black circles) top silver layer and (gray circles) bottom dielectric layer for one of the $M=1000$ effective electrons in the parallel-plate silver diel $_{\text {silver }}$ loaded waveguide. (a) Spacecharge effects are not considered. (b) Space-charge effects are considered.

(emitting secondary electrons) and against the bottom dielectric layer (also emitting secondary electrons). Thus, the dielectric sheet charges positively (as shown in Fig. 8). When the static field becomes intense enough, the positively charged dielectric material attracts effective electrons toward the bottom dielectric layer. Because resonance is lost, electrons are absorbed in these collisions, as low SEY values at the metallic plate from RF cycle 40 make it evident in Fig. 10(a) and (b). The individual populations $N_{i}$ diminish after these collisions. Thus, when they collide again against the bottom dielectric layer with high kinetic energies [as shown in Fig. 10(a) and (b)], fewer electrons are now emitted from the dielectric material (and the $E_{\mathrm{dc}}$ static field does not increase as much as in the previous impacts). The rate at which electrons are absorbed in the metallic layer is higher than the rate at which electrons are emitted from the dielectric bottom sheet. This results in a decreasing population 


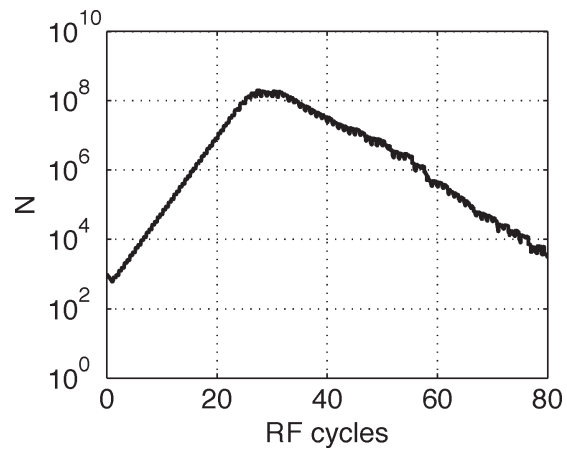

Fig. 11. Time evolution of the number of electrons $N$ for the parallel-plate alumina-loaded waveguide.

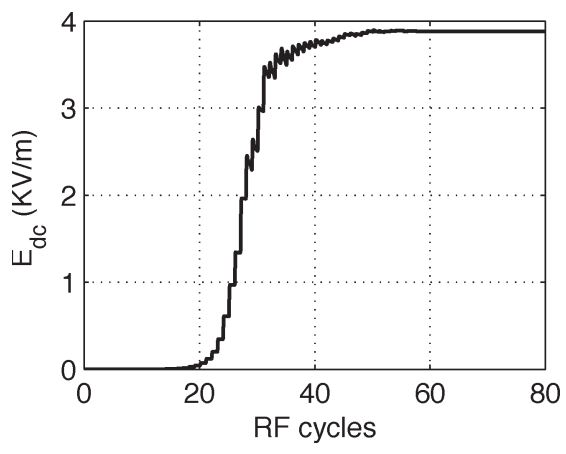

Fig. 12. $E_{\mathrm{dc}}$ static field time evolution for the silver parallel-plate aluminaloaded waveguide.

of electrons $N$ (until it finally disappears completely), whereas the dielectric layer remains finally charged at a constant value.

If space-charge effects are not neglected in a dielectricloaded waveguide like the one being studied, two saturating mechanisms affect simultaneously the electron discharge (and thus, the $M=1000$ effective electrons interact with one another not only through the dc static field but also through the space-charge sheet, accounting for space-charge effects). As can be noticed in Figs. 7 and 8, space charge basically constitutes a slowing factor in the extinction of the discharge. The electron bunch is also attracted by the positive $E_{\mathrm{dc}}$ static field as effective electrons are. Therefore, its main contribution to the development of the discharge is to disturb effective electrons whenever they are close to them by repelling them. For the particular conditions being studied, this implies that $N$ does not reach the same maximum value when space charge is neglected, the discharge finally shuts down 20 RF cycles later, and the $E_{\mathrm{dc}}$ static field saturates at a lower value.

\section{Parallel-Plate Alumina-Loaded Waveguide}

Finally, the realistic case of a parallel-plate waveguide loaded with a thin layer of alumina on top of the bottom silver surface is considered. Space-charge effects are accounted for. The SEY properties for alumina are given in Fig. 2 and Table I and have been obtained from [9]. For this case, the same parameters used in the previous section, with respect to the waveguide geometry and signal characteristics, are employed. Fig. 11 shows the time evolution of $N$ for this case, whereas Fig. 12 shows the evolution of the $E_{\mathrm{dc}}$ field. As explained in the previous section, the multipactor discharge finally disappears, whereas the dielectric sheet remains positively charged.

Future works using the model presented in this paper can include the presence of a second dielectric layer on top of the upper metallic surface in Fig. 1, as well as the consideration of real SEY measurements for the materials involved in the structure.

\section{CONCLUSION}

In this paper, a model to simulate multipactor discharges in a parallel-plate dielectric-loaded waveguide using multiple effective electrons is presented. Different geometries under a variety of working conditions have been investigated using the proposed technique in order to study the effects of the space charge and the static field which appears in dielectric-loaded structures. The performed simulations reveal that multipactor discharges in this type of dielectric-loaded waveguides turn off by themselves due to the appearance of time varying static fields. The space-charge main effect in the time evolution of the discharge is to slow down the switching off of the process. To the authors' knowledge, this is the first time that space-charge effects and time varying static electric fields in dielectricloaded waveguides have been simultaneously investigated in the context of multipactor discharges.

\section{REFERENCES}

[1] A. J. Hatch and H. B. Williams, "The secondary electron resonance mechanism of low-pressure high-frequency gas breakdown," J. Appl. Phys., vol. 25, no. 4, pp. 417-423, Apr. 1954.

[2] A. J. Hatch and H. B. Williams, "Multipacting modes of highfrequency gaseous breakdown," Phys. Rev., vol. 112, no. 3, pp. 681-685, Nov. 1958.

[3] J. R. M. Vaughan, "Multipactor," IEEE Trans. Electron Devices, vol. 35, no. 7, pp. 1172-1180, Jul. 1988.

[4] R. Kishek, Y. Y. Lau, and R. M. Gilgenbach, "Temporal evolution of multipactor discharge," in Proc. Part. Accel. Conf., Dallas, TX, May 1995, pp. 1599-1601.

[5] A. M. Pérez, C. Tienda, C. Vicente, A. Coves, G. Torregrosa, B. Gimeno, R. Barco, V. E. Boria, and D. Raboso, "Multipactor analysis in coaxial waveguides for satellite applications using frequency-domain methods," in Proc. IEEE MTT-S Int. Microw. Symp., San Francisco, CA, Jun. 2006, pp. $1045-1048$.

[6] C. Vicente, M. Mattes, D. Wolk, B. Mottet, H. L. Hartnagel, J. R. Mosig, and D. Raboso, "Multipactor breakdown prediction in rectangular waveguide based components," in Proc. IEEE MTT-S Int. Microw. Symp., Long Beach, CA, Jun. 2005, pp. 1055-1058.

[7] J. de Lara, F. Pérez, M. Alfonseca, L. Galán, I. Montero, E. Roman, and D. R. Garcia-Baquero, "Multipactor prediction for on-board spacecraft RF equipment with the MEST software tool," IEEE Trans. Plasma Sci., vol. 32, no. 2, pp. 476-484, Apr. 2006.

[8] R. A. Kishek and Y. Y. Lau, "Multipactor discharge on a dielectric," Phys. Rev. Lett., vol. 80, no. 1, pp. 193-196, Jan. 1998.

[9] R. A. Kishek, Y. Y. Lau, L. K. Ang, A. Valfells, and R. M. Gilgenbach, "Multipactor discharge on metals and dielectrics: Historical review and recent theories," Phys. Plasmas, vol. 5, no. 5, pp. 2120-2126, May 1998.

[10] H. C. Kim and J. P. Verboncoeur, "Modeling RF window breakdown: From vacuum multipactor to RF plasma," IEEE Trans. Dielectr. Electr. Insul., vol. 14, no. 4, pp. 766-773, Aug. 2007.

[11] J. G. Power, W. Gai, S. H. Gold, A. K. Kinkead, R. Konecny, C. Jing, W. Liu, and Z. Yusof, "Observation of multipactor in an alumina-based dielectric-loaded accelerating structure," Phys. Rev. Lett., vol. 92, no. 16, pp. 164801 1-164801 4, Apr. 2004.

[12] C. Jing, W. Gai, J. G. Power, R. Konecny, S. H. Gold, W. Liu, and A. K. Kinkead, "High-power RF tests on X-band dielectric-loaded accelerating structures," IEEE Trans. Plasma Sci., vol. 33, no. 4, pp. 1155-1160, Aug. 2005. 
[13] L. Wu and L. K. Ang, "Multipactor discharge in a dielectric-loaded accelerating structure," Phys. Plasmas, vol. 14, no. 1, pp. 013 105-1013 105-11, Jan. 2007.

[14] G. Torregrosa, A. Coves, C. P. Vicente, A. M. Pérez, B. Gimeno, and V. E. Boria, "Time evolution of an electron discharge in a parallel-plate dielectric-loaded waveguide," IEEE Electron Device Lett., vol. 27, no. 7, pp. 629-631, Jul. 2006.

[15] L.-K. Ang, Y. Y. Lau, R. A. Kishek, and R. M. Gilgenbach, "Power deposited on a dielectric by multipactor," IEEE Trans. Plasma Sci., vol. 26, no. 3, pp. 290-295, Jun. 1998.

[16] R. Seviour, "The role of elastic and inelastic electron reflection in multipactor discharges," IEEE Trans. Electron Devices, vol. 52, no. 8, pp. 1927-1930, Aug. 2005.

[17] C. Vicente, M. Mattes, D. Wolk, H. L. Hartnagel, J. R. Mosig, and D. Raboso, "Contribution to the RF breakdown in microwave devices and its prediction," in Proc. IEEE Int. Power Modul. Conf., Washington, DC, May 2006, pp. 22-27.

[18] E. Somersalo, P. Yl-Oijala, D. Proch, and J. Sarvas, "Computational methods for analyzing electron multipacting in RF structures," Part. Accel., vol. 59, no. 2, pp. 107-141, 1998.

[19] R. A. Kishek and Y. Y. Lau, "Interaction of multipactor discharge and RF circuit," Phys. Rev. Lett., vol. 75, no. 6, pp. 1218-1221, Aug. 1995.

[20] R. A. Kishek and Y. Y. Lau, "A novel phase focusing mechanism in multipactor discharge," Phys. Plasmas, vol. 3, no. 5, pp. 1481-1483, May 1996.

[21] S. Riyopoulos, "Multipactor saturation due to space-charge-induced debunching," Phys. Plasmas, vol. 4, no. 5, pp. 1448-1462, May 1997.

[22] A. Valfells, L. K. Ang, Y. Y. Lau, and R. M. Gilgenbach, "Effects of an external magnetic field, and of oblique radio-frequency electric fields on multipactor discharge on a dielectric," Phys. Plasmas, vol. 7, no. 2, pp. $750-757$, Feb. 2000

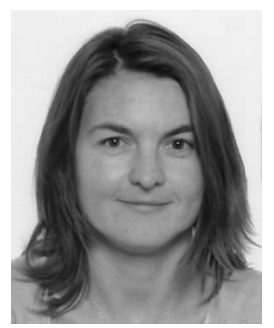

Ángela Coves (S'04-M'05) was born in Elche (Alicante), Spain, on May 20, 1976. She received the Licenciado degree in physics and the Ph.D. degree from the Universidad de Valencia, Valencia, Spain, in 1999 and 2004, respectively.

Since 2001, she has been a Lecturer with the Área de Teoría de la Señal y Comunicaciones, Universidad Miguel Hernández, Elche. Her current research interests include the study of electron discharges in microwave components and computer-aided techniques of the analysis of microwave passive components, waveguide structures, and diffraction gratings.

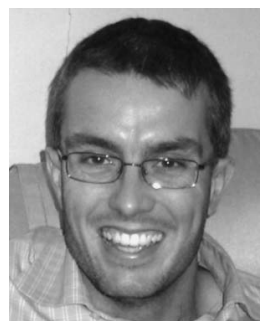

Germán Torregrosa-Penalva (S'00-M'05) was born in Novelda (Alicante), Spain, in 1976. He received the Ingeniero de Telecomunicación degree and the Ph.D. degree from the Universidad Politécnica de Madrid, Madrid, Spain, in 1999 and 2004, respectively.

In October 2002, he joined the Área de Teoría de la Señal y Comunicaciones, Universidad Miguel Hernández, Elche, Spain, where he is currently a Research and Teaching Assistant. His research interests include the study of electron discharges in microwave components, the thermal and electrical characterization and the equivalent circuit modeling extraction of microwave power amplifiers, and the design of microwave circuits and subsystems.

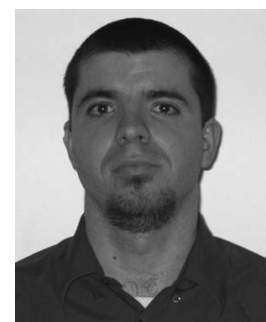

Carlos Vicente was born in Elche, Spain, in 1976. He received the Diploma degree in physics from the University of Valencia, Valencia, Spain, in 1999 and the Ph.D. degree in electrical engineering from the Institute of Microwave Engineering, Technical University of Darmstadt, Darmstadt, Germany.

From 1999 to the beginning of 2001, he was a Research Assistant with the Department of Theoretical Physics, University of Valencia. Since 2005, he has been with the Departamento de Comunicaciones, Universidad Politécnica de Valencia, Valencia. His current research concerns the design and analysis of passive microwave components with special emphasis in passive intermodulation, corona discharge, and multipaction effects.

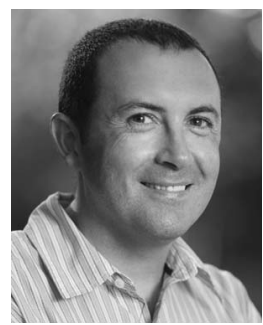

Benito Gimeno (M'01) was born in Valencia, Spain, on January 29, 1964. He received the Licenciado degree in physics and the Ph.D. degree from the Universidad de Valencia, Burjassot, Spain, in 1987 and 1992, respectively.

He was a Fellow with the Universidad de Valencia from 1987 to 1990. Since 1990, he has been an Assistant Professor with the Departamento de Física Aplicada y Electromagnetismo and the Instituto de Ciencia de Materiales, Universidad de Valencia, where he became an Associate Professor in 1997. During 1994 and 1995, he was with the European Space Research and Technology Center, European Space Agency, as a Research Fellow. In 2003, he obtained a fellowship from the Spanish Government for a short stay (three months) at the Universita degli Studi di Pavia, Pavia, Italy, as a Visiting Scientific. His current research interests include computer-aided techniques for the analysis of microwave and millimeter-wave passive components for space applications, waveguides, and cavity structures including dielectric objects, electromagnetic bandgap structures, frequency selective surfaces, and nonlinear phenomena appearing in power microwave subsystems (multipactor and corona effects and passive intermodulation phenomena).

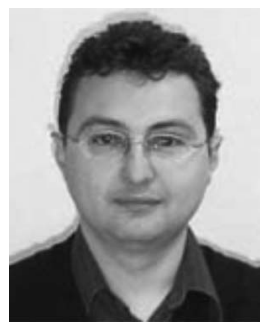

Vicente E. Boria (S'91-A'99-SM'02) was born in Valencia, Spain, on May 18, 1970. He received the "Ingeniero de Telecomunicación" degree (with first-class honors) and the "Doctor Ingeniero de Telecomunicación" degree from the Universidad Politécnica de Valencia, Valencia, in 1993 and 1997, respectively.

In 1993, he was with the "Departamento de Comunicaciones," Universidad Politécnica de Valencia, where he served as an Assistant Lecturer (in 1993-1995), Lecturer (in 1996-1997), and Associate Professor (in 1998-2002), and he is currently a Full Professor (since 2003). In 1995 and 1996, he was holding a Spanish Trainee Position with the European Space Research and Technology Center, European Space Agency, Noordwijk, The Netherlands, where he was involved in the area of EM analysis and design of passive waveguide devices. He is the author or coauthor of five chapters in technical textbooks, 50 papers in refereed international technical journals, and over 150 papers in international conference proceedings. His current research interests are focused on the analysis and automated design of passive components, left-handed and periodic structures, and on the simulation and measurement of power effects in passive waveguide systems.

Dr. Boria has been a member of the IEEE Microwave Theory and Techniques Society (IEEE MTT-S) and the IEEE Antennas and Propagation Society since 1992. He is a member of the Editorial Boards of the IEEE TRANSACTIONS on Microwave Theory and Techniques, IEEE Microwave AND Wireless Components LeTters, Proceeding of the IEE (Microwaves, Antennas, and Propagation), and IEE Electronics Letters and Radio Science. He is also a member of the Technical Committees of the IEEE-MTT International Microwave Symposium and the European Microwave Conference. He was the recipient of the 1993 First National Prize of Telecommunication Engineering Studies ("Ministerio de Educación y Ciencia"). He was also the recipient of the 2001 Social Council of Universidad Politécnica de Valencia First Research Prize for his outstanding activity during the period of 1995-2000. 